\title{
Processing of Alumina Nano-films
}

\author{
P. Mach, T. Rozkošný \\ Department of Electrotechnology, Faculty of Electrical Engineering, Czech Technical University in Prague, \\ Prague, Czech Republic \\ mach@fel.cvut.cz
}

\begin{abstract}
There are different technologies for processing of alumina thin films. It has been found that only some of them are usable for reliable and reproducible processing of nano-films with the thickness of 2 through $3 \mathrm{~nm}$. Such the films are used for fabrication of tunneling junctions with high sensitivity to humidity, which can be used for fabrication of humidity sensors. The alumina films are mostly prepared on evaporated or sputtered aluminum electrode by oxidization. Quality of tunneling junctions with alumina tunneling barrier fabricated of air-oxidized, oxygen-oxidized and oxygen plasma-oxidized Al electrodes has been examined by the measurement of resistances and capacitances of the junctions. Sensitivity of the junctions to external humidity has been examined, too. It has been found that the best technology for fabrication of alumina nano-films is plasmatic oxidation of aluminum evaporated thin films. It has been also found that the alumina nano-thin films are very sensitive to humidity.
\end{abstract}

\section{INTRODUCTION}

Nanotechnology is a new level of technology, which gives new possibilities in processing of materials and their exploitation. There are many different definitions of nanotechnology. Very often it is possible to meet with the definition that nanotechnology is such a type of technology, where one or more dimensions of a final product are in nanometers. More sophisticated definition is this one: "Nanotechnology is technology, where it is accepted by a production engineer, that it exists also quantum mechanics". It is necessary to involve a new principle into products fabricated by nanotechnology.

There are many different methods for preparation of alumina thin films. Typical vacuum technologies of processing of alumina thin films are PVD (physical vapor deposition) and CVD (chemical vapor deposition) [1], [2]. PVD process is usually carried out by sputtering of alumina target in Ar, CVD process is based on sputtering of $\mathrm{Al}$ target in a mixture of $\mathrm{Ar}+\mathrm{O}_{2}$. Another ways of fabrication of alumina films are galvanic oxidation, chemical oxidation and others [3]. It has been found that these technologies do not satisfy the requests for fabrication of tunneling barriers with the thickness of 2 through $3 \mathrm{~nm}$ by reason of too fast grow of the films. Therefore other technologies have been investigated.

There are many possible applications of alumina thin films with very low thickness. They are used as humidity-sensitive layers in humidity sensors, as barriers in inelastic electron tunneling spectroscopy, as barriers in tunneling junctions with unique properties e.g. junctions of a Josephson type, which can be used as basic cells of superconducting memories.

\section{Properties of A tUNNeling JunCtion}

Tunneling is a process joined with the fact that an electron has, instead others, also wave properties. These properties can be expressed, according to the Schrödinger equation, by a wave function. Transmission coefficient of an electron through an energetic barrier can be described by a formula:

$T \approx e^{-2 \chi L}$

Where

$\chi=\sqrt{\frac{2 m\left(E_{p 0}-E\right)}{\hbar^{2}}}$

Here $T$... coefficient of transmission of an electron, $m$... mass of an electron, $L \ldots$ thickness of the barrier, 
$E \ldots$ potential energy of an electron, $E_{p 0} \ldots$ potential energy of the barrier.

Tunneling is used in many applications. There are fabricated tunneling diodes with very short time of switching (e.g. 5 ps), the surfaces of materials are investigated by the use of scanning electron tunneling microscopy (STEM), and the principle of tunneling is also used in highly sensitive sensors of cosmic irradiation. Tunneling can also occur if the current is flowing between two metal contacts separated by an insulating barrier and this mechanism of conductivity influences also quality of adhesive conductive joints.

Tunneling of an electron through an insulating barrier can be joined with the loss of its energy - this type of tunneling is named inelastic or the electron can pass through the barrier without the energy loss - this type is named elastic tunneling. The current vs. voltage characteristics of the elastic tunneling is only weakly nonlinear, the $\mathrm{C}-\mathrm{V}$ characteristics of the inelastic tunneling is strongly nonlinear.

If it is accomplished a condition

$$
\frac{h \cdot v}{e}>>U
$$

the tunneling current, as a result of the elastic tunneling, can be described by the equation:

$$
I=\alpha \cdot\left(U+\gamma \cdot U^{3}\right)
$$

Where $h \ldots$ Planck constant, $v \ldots$ wave number, $e \ldots$ charge of an electron, $U \ldots$ applied voltage, $I \ldots$ tunneling current, $\alpha, \gamma \ldots$ constants, $\gamma \ll \alpha$.

If it is accomplished a condition

$$
U>\frac{h \cdot v}{e} \quad,
$$

the tunneling can be elastic or inelastic. The current can be calculated according to the formula of Fowler and Nordheim:

$$
\ln \frac{I}{U^{2}}=A+\frac{B \cdot h \cdot v \cdot L}{e \cdot U}
$$

Where $A, B \quad \ldots$ constants, $L \ldots$ thickness of an insulating barrier.

Resistance of an tunneling junction consisting of two metal electrodes separated by the insulating barrier strongly depends on the thickness of this barrier. This dependence can be described by the equation:

$$
R \approx \exp (L / D)
$$

Where $R$ resistance of the tunneling junction, $D$... constant, $D \approx 0.1$ through $0.7 \mathrm{~nm}$.

If the thickness $L$ of the insulating barrier will be increased $n$-times, then the value of the resistance of the tunneling junction will increase according to the formula:

$$
R_{n}=R_{0} \cdot[\exp (L / D)]^{n-1}
$$

Where $R_{n} \ldots$ final resistance of the tunneling junction, $R_{0} \ldots$ starting resistance of the tunneling junction.

Thickness $L$ of the insulating barrier of the tunneling junction is usually 2 through $3 \mathrm{~nm}$. For $L=$ $2 \mathrm{~nm}$ and $D=0.2 \mathrm{~nm}$ the resistance of the junction will depend on an increase of the thickness of the barrier as follows:

$$
R_{n}=R_{0} \cdot[\exp 10]^{n-1}=R_{0} \cdot\left(2.2 \cdot 10^{4}\right)^{n-1}
$$

The formula (9) shows very strong dependence of the resistance of the tunneling junction on the thickness of the insulating barrier.

\section{TECHNOLOGY OF FABRICATION OF THE TUNNELING JUNCTION}

The structure of the tunneling junction is shown in Fig. 1.

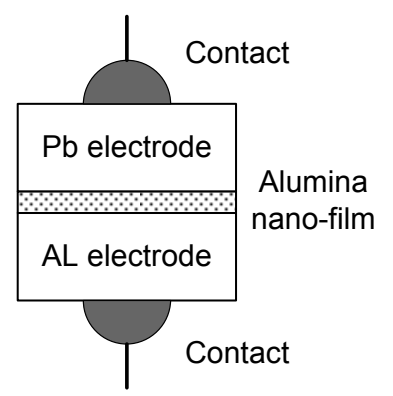

Fig. 1 Structure of the tunneling junction

The basic steps for fabrication of the tunneling junction are shown in Fig. 2.

Step A: evaporation or sputtering of the bottom electrode. The thickness of this electrode is not 
critical; usually the thickness 300 through $500 \mathrm{~nm}$ is used.

Step B: fabrication of the alumina nano-film. The technologies have been already mentioned and will be

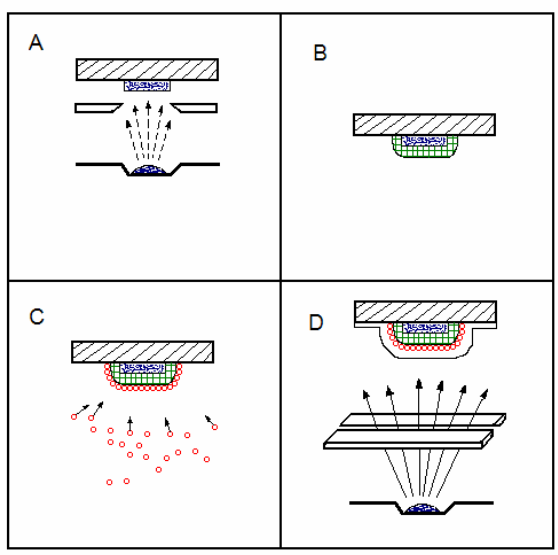

Fig. 2 Four steps of fabrication of the tunneling junction

discussed in a following part of the paper.

Step $C$ : doping of the tunneling junction by molecules of other material - this step is used if the junctions are prepared for spectroscopy, the junctions intended for humidity sensors are fabricated without this step.

Step D: evaporation of the top electrode of the tunneling junction. This electrode must not be sputtered, because the nano-film is very sensitive to an electrical discharge, which occurs during the sputtering process. The thickness of this electrode is not critical; usually the thickness 300 through $500 \mathrm{~nm}$ is used.

\section{TECHNOLOGY OF FABRICATION OF THE ALUMINA NANO-FILMS}

Alumina nano-films used as tunneling barriers of the junctions having aluminum bottom electrodes are usually fabricated by oxidization of these electrodes. This technology offers the nano-films of high homogeneity and makes their fabrication with high repeatibility possible. Usability of three types of technologies for preparation of alumina nano-films has been tested. The tunneling junctions have been fabricated with the barriers prepared by air oxidization of the bottom electrode, by oxidization of this electrode in oxygen and by plasmatic oxidization of the basic electrode.

Air oxidized nano-films have been prepared by the following way: after evaporation of the bottom Al electrode the specimen has been located inside a flow box with a dust filter. Oxidization has been carried out by air for $10 \mathrm{~min}, 1 \mathrm{~h}$, and $3 \mathrm{~h}$.

Air oxidization of the bottom electrode at the higher temperature has also been tested. The substrates with evaporated bottom electrodes have been located inside an oven for $10 \mathrm{~min}$; the temperature has been $100{ }^{\circ} \mathrm{C}$.

Oxidization of the bottom aluminum electrode in oxygen has been carried out inside the vacuum bell jar. After evaporation of this electrode the pumping of the bell jar has been stopped, and oxygen has been let in the bell jar. The pressure of oxygen has been $130 \mathrm{mPa}$. The time of oxidization has been 1,16 and $30 \mathrm{~h}$.

Plasmatic oxidization of the bottom electrode has been carried out in arrangement shown in Fig. 3.
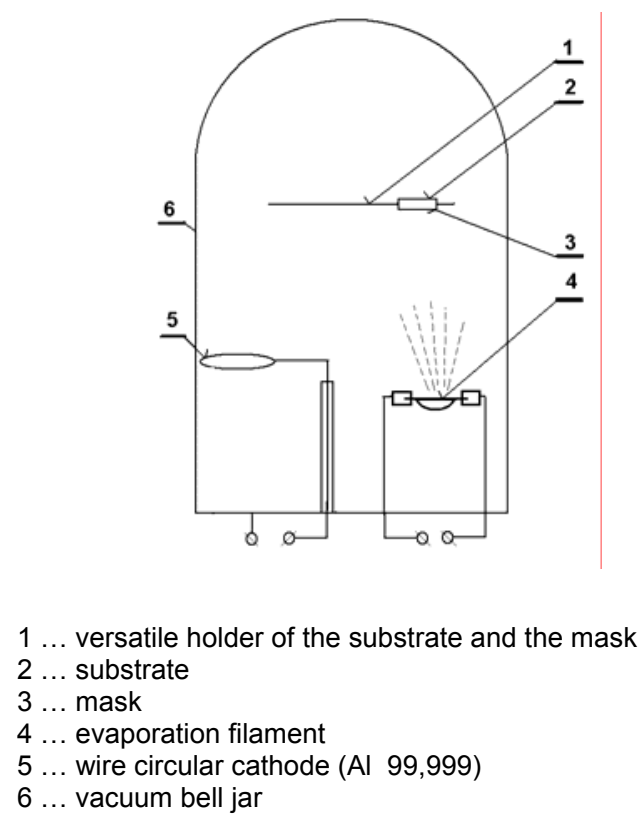

Fig. 3 Fabrication of the alumina nano-film by plasmatic oxidation

After evaporation of the bottom electrode the holder with the substrate has been turned to be the substrate approximately over the centre of the circular aluminum cathode fabricated of the wire with the diameter of $2.5 \mathrm{~mm}$. The desk valve has been closed and the pumping of the recipient by the diffusion 
pump has been stopped. The bell jar has been pumped by the rotary pump only. Then the needle valve for inflow oxygen has been opened and the flow rate of oxygen has been set up to be reached the pressure of 4 $\mathrm{Pa}$ inside the bell jar. Then the adjustable dc voltage has been connected between the cathode and the bell jar (the bell jar has been grounded). The current flowing through the discharge has been adjusted on the value of $2.5 \mathrm{~mA}$. It has been found that such the value of the current gives the most reliable results with the highest repeatability. The oxidization has been carried out 10, 20 and 30 minutes.

After the fabrication of the alumina barrier the junction has been created by evaporation of the top aluminum or led electrode across the bottom one. The width of the bottom as well as the top electrode has been $0.7 \mathrm{~mm}$, the thickness $300 \mathrm{~nm}$. The material of the electrodes has been aluminum (99.999). The junctions have been fabricated on glass substrates.

\section{EXPERIMENTAL RESULTS}

The resistance and capacitance of the junctions have been measured. The equipment Tesla BM-RLC 420 Meter has been used for the measurement. This equipment makes possible the measurement of the resistance with accuracy of $1 \%$ of the full range and capacitance of the $2 \%$ of the full range. The measuring frequency of the capacitance is $1 \mathrm{kHz}, 20$ $\mathrm{kHz}$ and $100 \mathrm{kHz}$. The frequency $1 \mathrm{kHz}$ has been used for our measurements.

It has been found that the variance coefficient of the air-oxidized films is too high (higher than 1.8). The reason of such the high variance has been quality of the air. The oxidation has been carried out in Prague, where it is possible to assume higher contents of NOx and SOx. Therefore this technology has had to be rejected.

The resistances of the alumina nano-films fabricated by oxidation of the aluminum electrode in air at the temperature of $100{ }^{\circ} \mathrm{C}$ have been very high and the variance coefficient of these films has been comparable with the coefficient of the films prepared by oxidation in air. The growth of these films has been very fast, therefore, with respect to the formulas (7) through (9), this technology is not proper for fabrication of the tunneling films.
The nano-films prepared by oxidation in oxygen have the acceptable variation coefficient $(0.3)$, but the growth of the films has been too slow. The requested values of the junctions, 100 through $1000 \Omega$, have been achieved after 30 hours of oxidation.

Oxidation in oxygen plasma has been found as the best way how to prepare, by oxidation of the basic electrode, the alumina nano-films with requested values of resistance and during the short time. The variation coefficient of the resistances of fabricated junctions has been in the range 0.2 through 0.3 , it is possible to control the time of oxidation by control of the current flowing through the oxygen plasma.

An example of values of the resistances and capacitances of the junctions fabricated by plasmatic oxidation is shown in Tab. 1. The values of capacitances are very high with respect to the area of the junction $\left(\sim 0.5 \mathrm{~mm}^{2}\right)$. The reason of the high capacitance is very low thickness of the insulating barrier. The breaking voltage of the junctions is approximately $2.5 \mathrm{~V}$.

\begin{tabular}{|c|l|r|r|r|r|r|r|r|r|r|}
\hline \multicolumn{2}{|c|}{ Specimen Junction } & 1 & 2 & 3 & 4 & 5 & 6 & 7 & 8 \\
\hline \multirow{2}{*}{$\mathbf{1}$} & $\mathbf{R}[\Omega]$ & 1397 & 1331 & 1378 & 1220 & 1230 & 1309 & 1470 & 1305 \\
& $\mathbf{C}[\mathrm{nF}]$ & 17.7 & 17.9 & 14.9 & 19.1 & 15.5 & 17.5 & 21.4 & 12.9 \\
\hline \multirow{2}{*}{$\mathbf{2}$} & $\mathbf{R}[\Omega]$ & 194.1 & 199.1 & 191.3 & 174.6 & 191.3 & 214 & 208 & 198 \\
\cline { 2 - 11 } & $\mathbf{C}[\mathrm{nF}]$ & 17.1 & 18.9 & 18.1 & 17.5 & 18.3 & 17.2 & 20.1 & 19.2 \\
\hline \multirow{2}{*}{$\mathbf{3}$} & $\mathbf{R}[\Omega]$ & 194.1 & 185.8 & 195.3 & 180.2 & 184.3 & 165.9 & 201.1 & 197.5 \\
\cline { 2 - 11 } & $\mathbf{C}[\mathrm{nF}]$ & 16.6 & 19.4 & 16.7 & 16.2 & 18.9 & 19.7 & 21.1 & 21 \\
\hline \multirow{2}{*}{$\mathbf{4}$} & $\mathbf{R}[\Omega]$ & 971 & 805 & 938 & 891 & 935 & 876 & 920 & 881 \\
& $\mathbf{C}[\mathrm{nF}]$ & 17 & 19.5 & 19.4 & 20.1 & 20.8 & 20.2 & 18.9 & 25.8 \\
\hline \multirow{2}{*}{$\mathbf{5}$} & $\mathbf{R}[\Omega]$ & 170.6 & 180 & 183.7 & 163.5 & 191.2 & 177.6 & 185.4 & 193.8 \\
& $\mathbf{C}[\mathrm{nF}]$ & 19.5 & 19 & 18.5 & 18.9 & 18.4 & 19.3 & 16.9 & 18.1 \\
\hline
\end{tabular}

Tab. 1 Values of the resistances and capacitances of one group of tunneling junctions fabricated by plasmatic oxidation of the bottom $\mathrm{Al}$ electrode

\section{AgING OF ALUMina NANO-FILMS}

The tunneling junctions fabricated by plasmatic oxidation of the bottom electrode have been aged in air and in a dry dessicator. Changes of the resistances and capacitances of the junctions aged in air are shown in Fig. 4, changes of the resistances and capacitances of junctions aged in a dry dessicator are shown in Fig. 5.

It has been found that aging of the junctions in air will cause significant changes of their resistance after some hours. Aging of the junctions of the same type and comparable resistance in a dry dessicator will cause higher changes of the resistances after more than 
100 hours. That means that the main reason of the change of the resistivity of the junctions is humidity. It is assumed that molecules of water diffuse into the junction through the top electrode and create oxides $\mathrm{Al}_{2} \mathrm{O}_{3}$ or hydrides $\mathrm{Al}(\mathrm{OH})_{3}$ inside the junction. The thickness of the insulating barrier increases. With respect to the extreme sensitivity of the junction resistance to the thickness of the insulating barrier the resistance dramatically increases.

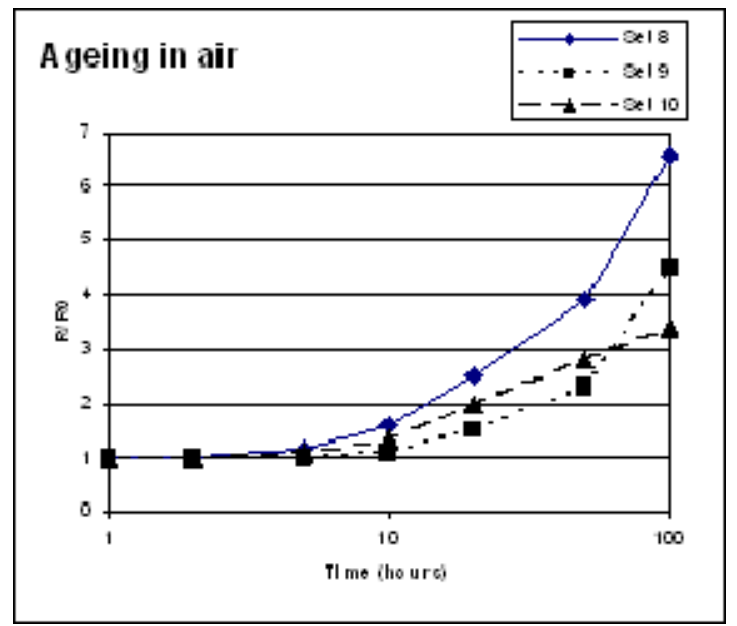

Fig. 4 Changes of the resistances of selected groups of the tunneling junctions. The junctions have been aged in air

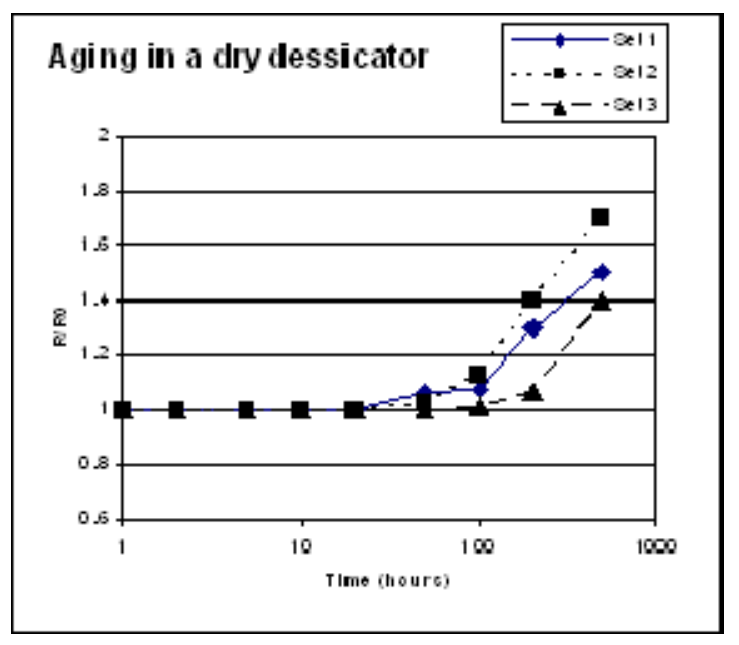

Fig. 4 Changes of the resistances of selected groups of the tunneling junctions. The junctions have been aged in a dry dessicator

\section{Conclusions}

Three different technologies for fabrication of the alumina nano-films have been investigated. It has been found that technology, which affords optimum results, is plasmatic oxidation of the aluminum electrode. The quality of the films has been examined by the measurement of the quality of the tunneling junctions. whose insulating barrier has been the alumina nano-film. It has been found that the plasmatic technology makes possible fabrication of the junctions with requested values of the resistance. The capacitance of the junctions is, with respect to the thickness of the insulating barrier, very high.

The junctions have been aged in air and in a dry dessicator. It has been found that the aging in air causes significant changes of the resistances of the junctions after some hours. The storing of the junctions in the dry dessicator has caused comparable changes after 100 hours. It has been found that the changes of the resistances of the junctions are caused by the diffusion of water molecules into the junctions. It has been confirmed that the tunneling junctions of this structure can be used as a very sensitive humidity sensors, which can be used in many applications.

\section{REFERENCES}

[1] http://www.ifm.liu.se/thinfilmprogram/projects/p3.htm

[2] E.S.K. Menon, I. Dutta, „Processing and characterization of alumina thin films on chemically vapor deposited diamond substrates for producing adherent metallizations“, J. of Mat. Res., pp. 565-577, 1999

[3] Jon M. Andersson et al, ,Microstructure of a-alumina thin films deposited at low temperatures on chromia template layers", J. Vac. Sci. Technol. A, Vol. 22, No. 1, Jan'Feb., pp. 117-121, 2004

\section{ACKNOWLEDGMENT}

The work has been supported by a research project $\mathrm{Nr}$. 34/05212/13113 "Diagnostics of materials". 